\title{
Evaluación tomográfica del cóndilo y fosa mandibular en el tratamiento de las maloclusiones Clase II y Clase III. Revisión de Literatura
}

Tomographical evaluation of the condyle and mandibular fossa in the treatment of Class II and Class III malocclusions. Literature Review.

\author{
Manuel Gustavo Chávez - Sevillano ${ }^{1, a ; 2, b}$, Guaracilei Maciel Vidigal Junior ${ }^{3, c}$, Cátia Cardoso Abdo \\ Quintão $^{4, c}$
}

\section{RESUMEN}

El tratamiento de varias maloclusiones esqueléticas en pacientes niños y jóvenes son realizadas aplicándose fuerzas ortopédicas, buscando alterar el crecimiento o abrir suturas en determinadas regiones anatómicas. El cóndilo $(\mathrm{CO})$ y la fosa mandibular (FM) son regiones que han sido sometidas a fuerzas intensas esperando su remodelación ósea como parte del tratamiento. Debido a los clásicos registros bidimensionales utilizados para evaluar las alteraciones morfológicas anatómicas, muchos resultados han sido controversiales. Con el uso de las tomografías y las modernas técnicas de superposiciones tomográficas, es posible identificar dichos cambios morfológicos de manera cuantitativa y cualitativa en las estructuras óseas. Se realizó una revisión de literatura integrada de las alteraciones morfológicas del CO y la FM evaluados por medio de la tomografía computarizada (TC) y la tomografía computarizada de haz cónico (TCHC) en pacientes con maloclusiones esqueléticas Clase II y Clase III que usaron aparatos con fuerzas ortopédicas. El objetivo fue identificar en la literatura los cambios morfológicos que ocurrieron en el $\mathrm{CO}$ y la FM después de aplicar los más aceptados protocolos de tratamientos de las respectivas maloclusiones. De esta manera nos permitirá eliminar factores intervinientes como distorsiones, superposiciones no deseadas de estructuras anatómicas y diversos errores de medición que tienen como característica los clásicos registros bidimensionales y que alteran la correcta información.

PALABRAS CLAVE: maloclusión; mandíbula; tomografía computarizada de haz cónico; imagen tridimensional.

\footnotetext{
Doctorado en Ortodoncia, Departamento de Odontología Preventiva y Comunitaria, Facultad de Odontología, Universidad del Estado de Rio de Janeiro.Rio de Janeiro, Brasil.

Departamento de Estomatología Pediátrica, Facultad de Odontología, Universidad Nacional Mayor de San Marcos. Lima, Perú

Departamento de Procedimientos Clínicos Integrados, Facultad de Odontología, Universidad del Estado de Rio de Janeiro. Rio de Janeiro, Brasil.

4 Departamento de Odontología Preventiva y Comunitaria. Facultad de Odontología, Universidad del Estado de Rio de Janeiro. Rio de Janeiro, Brasil

MSc. Estudiante ; ${ }^{b}$ Profesor; ${ }^{c}$ PhD. Profesor;
} 


\section{SUMMARY}

The treatment of various skeletal malocclusions in children and young' patients are performed applying orthopedic forces, seeking to alter growth or open sutures in certain anatomical regions. The condyle (CO) and mandibular fossa (MF) are regions that have been subjected to intense forces awaiting bone remodeling as part of the treatment. Due to the classic two-dimensional records used to assess anatomical morphological changes, many results have been controversial. With the use of tomography and modern tomographic superposition techniques, it is possible to identify these morphological changes quantitatively and qualitatively in bone structures. An integrated literature review of the morphological alterations of the $\mathrm{CO}$ and the MF was performed using Computed Tomography (CT) and Cone Beam Computed Tomography (CBCT) in patients with Class II and Class III skeletal malocclusions who used appliances with orthopedic forces. In the literature review, the objective was to identify morphological changes that occurred in the $\mathrm{CO}$ and the MF after applying the most accepted treatment protocols for the respective malocclusions. In this way, it will allow us to eliminate intervening factors such as distortions, unwanted overlaps of anatomical structures and various measurement errors that have the characteristic of two-dimensional classic records altering the correct information.

KEYWORDS: Malocclusion; mandible; cone-beam computed tomography and three-dimensional imaging.

\section{INTRODUCCION}

La maloclusión esquelética Clase II es una de las alteraciones con alta prevalencia en la población y representa aproximadamente un tercio de los problemas ortodóncicos (1). La etiología más común es la retrusión mandibular, por lo tanto, si el paciente está todavía en crecimiento, la principal terapia consiste en reubicar y adelantar la mandíbula, esperando un estímulo del crecimiento a nivel condilar (2). La maloclusión esquelética Clase III, la cual tiene etiología principalmente hereditaria combinada con factores ambientales (3), tiene como usual propuesta de tratamiento para pacientes niños y jóvenes el estímulo del crecimiento maxilar en dirección anterior. Por lo tanto, el uso de aparatos que aplican fuerzas buscando estimular el crecimiento de las bases óseas maxilar y mandibular es común en Ortodoncia.

Buscando identificar los efectos esqueléticos y dentarios de los aparatos ortopédicos, han sido reportados estudios donde utilizaron herramientas de evaluación bidimensional (2D) como las radiografías cefalométricas y tridimensional (3D) como la Resonancia Nuclear Magnética(4), la tomografía computarizada (TC) $(5,6)$, y la tomografía computarizada de haz cónico (TCHC) $(7,8,9,10)$.

La TC, en especial la TCHC, es considerada actualmente el "patrón de oro" como herramienta para identificar y evaluar las estructuras óseas en el diagnóstico 3D en Odontología. De esta manera, varios análisis que incluyen a la Articulación Temporomandibular (ATM) usando TC $(5,6)$ y TCHC $(8,9)$ han sido realizados en pacientes con maloclusión esquelética Clase II que usaron aparatos propulsores de la mandíbula. Similares herramientas de evaluación se han aplicado en estudios con pacientes que tenían maloclusión esquelética Clase III sobre los cuales realizaron modernos tratamientos para estimular el crecimiento maxilar(11,12,13) (figura 1).

Una manera moderna de realizar una investigación bibliográfica es por medio de una metodología integrada, la cual incluye diversas fuentes de datos los cuales mejoran la comprensión holística del tópico en interés. Incorpora además, una sistemática y rigurosa propuesta de los procesos, particularmente del análisis de los datos encontrados en los artículos (14).

El uso de aparatos propulsores de la mandíbula y la protracción maxilar son frecuentes tratamientos en pacientes en crecimiento con maloclusiones esqueléticas Clase II y Clase III respectivamente. Los efectos que estos aparatos causan sobre el cóndilo $(\mathrm{CO})$ y fosa mandibular (FM) no están aún bien esclarecidos tridimensionalmente (15), por lo que la presente revisión de literatura, busca realizar un análisis crítico de los estudios que muestran las 


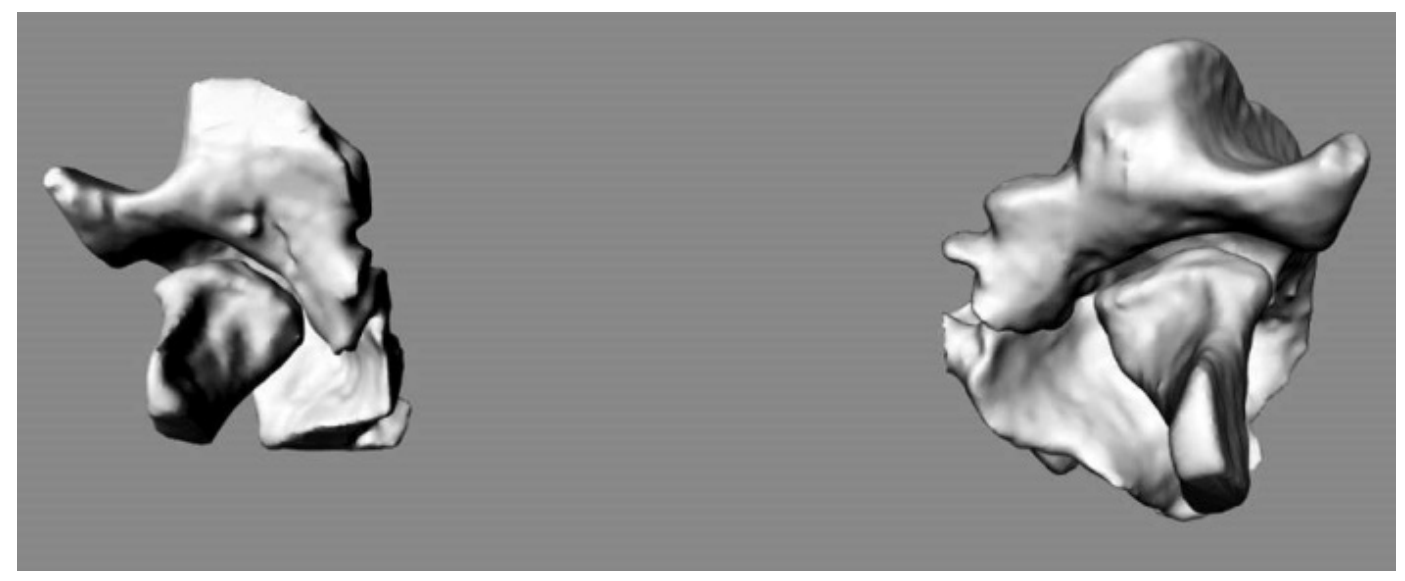

Figura 1. Cóndilo y fosa mandibular segmentada de una TCHC.

alteraciones del $\mathrm{CO}$ y FM por medio de TC y TCHC en pacientes con maloclusiones esqueléticas Clase II y Clase III que usaron aparatos con fuerzas ortopédicas.

\section{MATERIAL Y METODOS}

La búsqueda bibliográfica para esta revisión integrada de literatura fue realizada en las siguientes bases de datos: Medline via Pubmed, Scopus y Cochrane. Fueron usadas las siguientes palabras claves: "Malocclusion", "Mandible", Cone-Beam Computed Tomography" y "Three-Dimensional Imaging"; asociadas a los siguientes operadores boleanos: AND y OR, en Abril del 2019. En la investigación bibliográfica también fueron aplicados los siguientes filtros: revistas científicas odontológicas y artículos publicados en los últimos 20 años, seleccionándose 36 títulos de artículos. Seguidamente fueron usados los siguientes criterios de inclusión: estudios en humanos, pacientes con maloclusión de Angle Clase II división 1, pacientes con maloclusión de Angle Clase III, pacientes con relación esquelética Clase II y Clase III, uso de tomografías y evaluación del CO y FM. Fueron considerados como criterios de exclusión: uso de solo radiografías cefalométricas, pacientes con dentición decidua y presencia de síndromes craneofaciales.

\section{RESULTADOS}

Después de la aplicación de los criterios de inclusión y de exclusión fueron seleccionados 25 títulos para la lectura del texto completo. De estos artículos fueron seleccionados 13 estudios, los cuales nos proporcionaron resultados específicos sobre cambios morfológicos del CO y FM. Estos artículos fueron agrupados en base al tipo de maloclusión esquelética tratada (tabla 1 y tabla 2 ).

\section{Tratamiento de las maloclusiones esqueléticas en pacientes en crecimiento.}

Con relación al tratamiento de las maloclusiones esqueléticas Clase II, dentro de los estudios encontrados utilizando TC y TCHC en pacientes tratados con un aparato propulsor mandibular, son más frecuentes el uso del Aparato de Herbts (AH) $(5,8,16,17)$, y del aparato funcional Bloque gemelo (BG) $(18,9)$. Esto está relacionado con la efectividad encontrada en previos estudios de aquellos aparatos (19); como también fue encontrado un estudio donde se usó el aparato Forsus (6). Con relación al tratamiento de la maloclusión esquelética clase III, evaluada con TCHC, fue común encontrar el uso de anclaje esquelético con mini placas de titanio combinado con elásticos de Clase III para realizar la protracción maxilar $(7,11,12)$. Cevidanes et al., (7), validaron un determinado método de superposición de imágenes 3D de estructuras anatómicas. Para eso utilizaron 6 TCHCs de 3 pacientes con maloclusión esquelética Clase III los cuales fueron sometidos a un tratamiento de protracción maxilar con anclaje esquelético por medio de mini placas de titanio y el uso de elásticos clase III (20). Los pacientes tuvieron una edad promedio de 11,4 años. Se realizó la superposición de las tomografías registradas antes y después del tratamiento encontrándose un buen grado de reproducción del método, al identificarse desplazamientos y remodelaciones óseas en la maxila y la mandíbula. 
Tabla 1. Efectos sobre el CO y FM en el tratamiento de la maloclusión esquelética Clase II

\begin{tabular}{|c|c|c|c|c|c|}
\hline Autor/Año & Resultado & G.C/G.T & Tiempo & Método & Edad \\
\hline Croft et al.1999 & $\begin{array}{l}\text { Crec.Post. del CO:2,1mm. } \\
\text { Despl. Post. e Inf. de la } \\
\text { FM:0,7mm }\end{array}$ & 40/40 (AH). & 11 meses & $\mathrm{TC}$ & 7-10 a. \\
\hline Arici Et al.2008 & $\begin{array}{l}\text { Aumento de Volumen del CO } \\
\text { y FM } \\
\text { Despl. Post. del CO }\end{array}$ & 30/30 (Forsus). & 7 meses & $\mathrm{TC}$ & 12 a. $7 \mathrm{~m}$. \\
\hline Bowen et al.2013 & $\begin{array}{l}\text { Aumento en Altura del } \\
\text { CO:6,20mm } \\
\text { Sagital:7,22mm }\end{array}$ & 0/20 (BG). & 12 meses & $\mathrm{TCHC}$ & $10-13$ a. \\
\hline LeCornu et al.2013 & $\begin{array}{l}\text { Despl. Ant. del } \\
\text { CO Der: } 1,2 \mathrm{~mm} \\
\text { Izq: } 1,29 \mathrm{~mm} \\
\text { Despl. Ant. } \\
\text { FM Der: } 1,69 \mathrm{~mm} \\
\text { FM Izq: } 1,43 \mathrm{~mm} \text {. }\end{array}$ & $7 / 7(\mathrm{AH})$ & $\begin{array}{l}\text { GC: } 18,42 \text { meses } \\
\text { GT:11,42meses }\end{array}$ & $\mathrm{TCHC}$ & $\begin{array}{l}\text { GC: } 13,4 \text { a. } \\
\text { GT:13 a. }\end{array}$ \\
\hline Yildirim et al.2014 & Crec. Sup. y Post. del CO & 0/30 (BG). & 7,4 meses & $\mathrm{TCHC}$ & $\begin{array}{l}\text { H: } 12,83 \text { a. } \\
\text { M:12,5 a. }\end{array}$ \\
\hline Souki et al.2017 & $\begin{array}{l}\text { Crec Sup y Post } \\
\text { del CO. GT:3,5mm } \\
\text { GC: } 2 \mathrm{~mm}\end{array}$ & 25/25 (AH). & 8 meses & $\mathrm{TCHC}$ & 12-16años. \\
\hline Batista et al.2017 & Crec Sup del CO:8,4mm & 20/20 (AH). & 12 meses & $\mathrm{TCHC}$ & 10-14años. \\
\hline Lima, 2016 & $\begin{array}{l}\text { Crec. del CO } \\
\text { Der BG: } 6,12 \mathrm{~mm} \text {. } \\
\text { Izq BG: } 6,34 \mathrm{~mm} . \\
\text { Der AH: } 7,01 \mathrm{~mm} \text {. } \\
\text { Izq AH: } 6,84 \mathrm{~mm} .\end{array}$ & 0/22AH(7),BG(15) & 12 meses & $\mathrm{TCHC}$ & $\begin{array}{l}\text { BG, H: 12,3a. } \\
\text { M: 11,9a. } \\
\text { AH, H: 13,6a. } \\
\text { M: 12,3a. }\end{array}$ \\
\hline
\end{tabular}

GC: Grupo Control; GT: Grupo de Trabajo; AH: Aparato de Herbts; BG: Bloque Gemelo; CO:Cóndilo; FM: Fosa Mandibular; TC:Tomografia Computarizada; Crec:Crecimiento; TCHC:Tomografía Computarizada de Haz Cónico; Ant: Dirección Anterior; Post: Dirección Posterior; Sup: Dirección Superior; Inf: Dirección Inferior; Der: Derecho; Izq: Izquierdo;Despl: Desplazamiento; a: años; m: meses, H: Hombres; M: Mujeres.

\section{Efectos sobre el Cóndilo y la Fosa Mandibular en el tratamiento de la maloclusión esquelética Clase II analizados con TC y TCHC}

Cuando fue usado el $\mathrm{AH}$ como protocolo de tratamiento, Souki et al., (16) encontraron 3,5mm de crecimiento posterior del $\mathrm{CO}$ y Croft et al., (5) registraron $2,1 \mathrm{~mm}$ en similar sentido. Se puede atribuir esa diferencia a la falta de homogeneidad etaria de los grupos analizados. En el primer estudio estuvieron en la pubertad y en el segundo estudio en la etapa de pre pubertad. Es importante resaltar que ambos trabajos tuvieron grupo control, Souki et al., (16) usaron un grupo control con tratamiento ortodóncico sin fuerzas ortopédicas y Croft et al., (5) utilizaron un grupo control sin tratamiento alguno. Por lo tanto, es posible que en el resultado del segundo estudio este descartado la influencia del crecimiento natural del paciente y el resultado se deba exclusivamente al AH. Otro factor que pudo haber influenciado en la diferencia de resultados es que Souki et al., (16) utilizaron la TCHC y un método de superposición tridimensional (10) y Croft et al., (5) usaron la TC y un método de superposición bidimensional basado en la cefalometría.

Souki et al., (16), también encontraron un crecimiento en dirección superior del $\mathrm{CO}$ con el uso del $\mathrm{AH}$, lo que concuerda con los hallazgos de Batista et al., (17), quienes comparando el uso del $\mathrm{AH}$ de manera convencional con otro $\mathrm{AH}$ con anclaje esquelético mandibular registraron valores de crecimiento del $\mathrm{CO}$ de $8,4 \mathrm{~mm}$.

LeCornu et al., (8), en un estudio prospectivo utilizando el $\mathrm{AH}$ encontraron valores de 
Tabla 2. Efectos sobre el CO y FM en el tratamiento de la maloclusión esquelética Clase III

\begin{tabular}{|c|c|c|c|c|c|}
\hline Autor/Año & Resultado & G.C/G.T & Tiempo & Método & Edad \\
\hline Cevidanes et al. 2007 & $\begin{array}{l}\text { Despl. Post. del CO. } \\
\text { Bimax:0,77mm. } \\
\text { Unimax: 0,70mm. }\end{array}$ & $\begin{array}{l}\text { 0/21 (Tx. Qx. } \\
\text { Avance Max.) } \\
\text { Tx.Qx. Setback } \\
\text { mandibular. }\end{array}$ & 7 dias & TCHC & $21,6+/-7,9$ a. \\
\hline Cevidanes et al.2009 & $\begin{array}{l}\text { Validación del método de } \\
\text { Superposición. }\end{array}$ & $\begin{array}{l}\text { 0/3 (Miniplacas } \\
\text { +Elásticos Clase III). }\end{array}$ & $\begin{array}{l}\text { No } \\
\text { determinado }\end{array}$ & TCHC & $11,4 \mathrm{a}$. \\
\hline De Clerck et al.2012 & $\begin{array}{l}\text { Despl. Post. del CO: } \\
\text { 2,07mm. } \\
\text { Remod. FM. } \\
\text { Ant. Aposic.:1,38mm } \\
\text { Post. Reabs.:1,34mm. }\end{array}$ & $\begin{array}{l}\text { 0/25 (Miniplacas } \\
\text { +Elásticos Clase III). }\end{array}$ & $1,2 \mathrm{a}$. & $\mathrm{TCHC}$ & $9-13$ a. \\
\hline Nguyen et al. 2014 & $\begin{array}{l}\text { Despl. Post. del } \\
\text { CO: } 2,6+/-1,5 \mathrm{~mm} \text {. }\end{array}$ & $\begin{array}{l}\text { 0/25 (Miniplacas } \\
\text { +Elásticos Clase III). }\end{array}$ & $12 \mathrm{~m}$ & $\mathrm{TCHC}$ & $9-13$ a. \\
\hline Yatabe et al.2017 & $\begin{array}{l}\text { Despl. Post. , Lateral e Inf. } \\
\text { de la FM del GC y el GT }\end{array}$ & $\begin{array}{l}\text { 24/19 } \\
\text { (Paladar Fisurado } \\
\text {-Miniplacas } \\
\text { +Elásticos Clase III). }\end{array}$ & $\begin{array}{l}\text { GT: } 18 \mathrm{~m} . \\
\text { GC: } 12 \mathrm{~m} .\end{array}$ & TCHC & $\begin{array}{l}\text { GT: } 11,8 \text { a. } \\
\text { GC: } 11,7 \text { a. }\end{array}$ \\
\hline
\end{tabular}

GC: Grupo Control; GT: Grupo de Trabajo; CO:Cóndilo; FM: Fosa Mandibular; Remod: Remodelación; Aposic: Aposición; Reabs: Reabsorción; Tx: Tratamiento; Qx: Quirúrgico; TCHC:Tomografía Computarizada de Haz Cónico; Bimax: Bimaxilar; Unimax: Unimaxilar; Ant: Dirección Anterior; Post:Dirección Posterior; Sup:Dirección Superior; Inf: Dirección Inferior; Despl: Desplazamiento; a: años; m:meses.

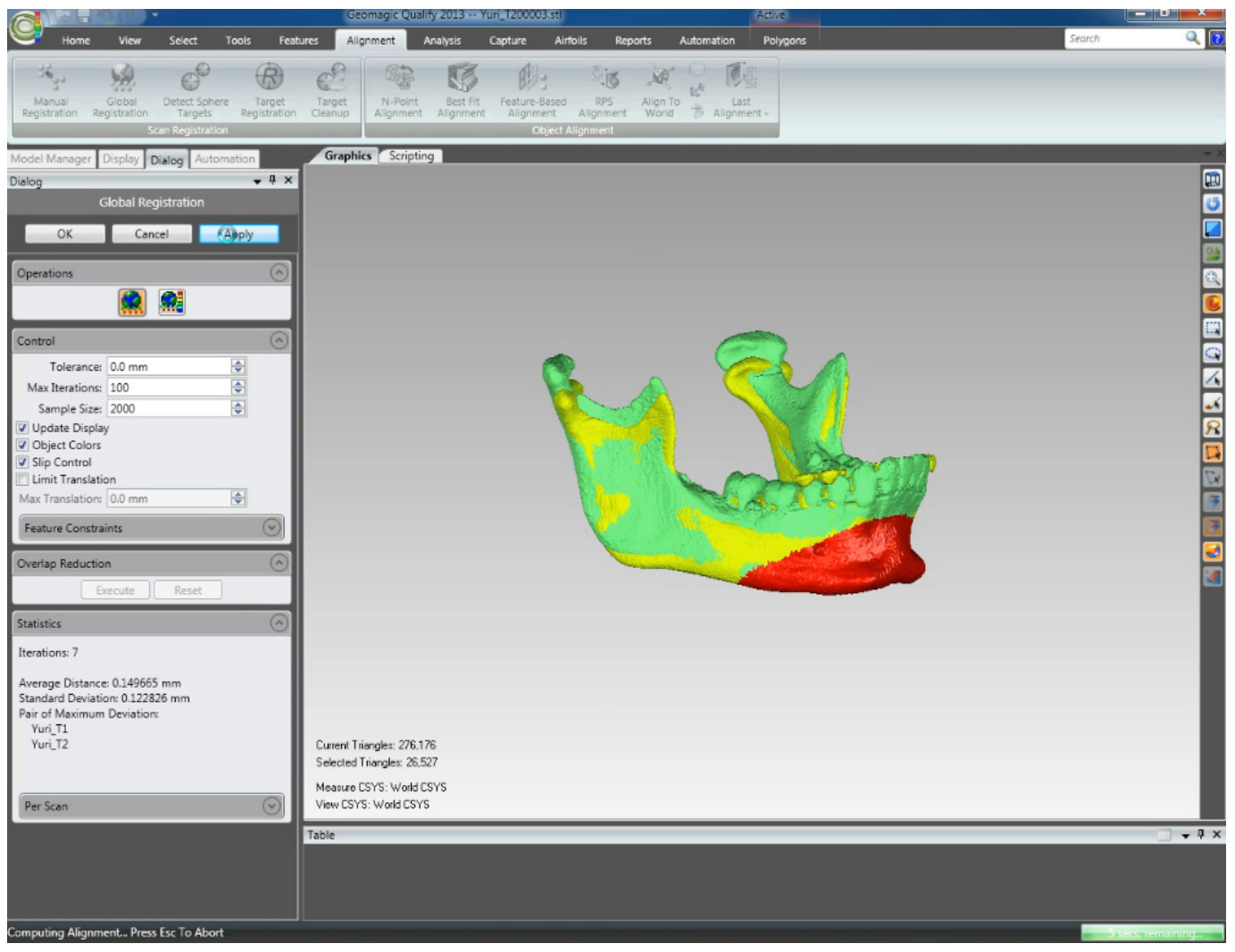

Figura 2. Superposición tomográfica con la técnica “best fit” (Software Geomagic Qualify 2013). 
desplazamiento anterior de $1,20 \mathrm{~mm}$ para el $\mathrm{CO}$ derecho y de $1,29 \mathrm{~mm}$ para el $\mathrm{CO}$ izquierdo. Se tuvo como grupo control a pacientes con elásticos Clase II, lo cual podría haber influenciado en estos resultados por la tendencia de los elásticos a llevar la mandíbula hacia adelante. En el mismo estudio fueron registrados desplazamientos en dirección anterior de la FM, siendo estos de 1,69mm. para la FM derecha y de $1,43 \mathrm{~mm}$ para la FM izquierda. Estos resultados de la FM son inconsistentes con los hallazgos de Croft et al., (5), quienes registraron desplazamientos de $0.7 \mathrm{~mm}$ en sentido posterior e inferior de la FM. Tal inconsistencia podría estar relacionado con la diferencia de edades promedios de las muestras, diferentes tipos de grupos control y técnicas de superposición (7) (21) (Tabla I).

Con el uso del aparato Forsus, Arici et al., (6) encontraron un aumento del volumen del $\mathrm{CO}$ y de la FM después de 7 meses de tratamiento. Fue utilizado un grupo control con pacientes sin tratar y con edad promedio de 12,7 años. Dicho aumento de volumen no fue significativo, por lo que el aparato Forsus no demostró tener un efecto relevante sobre el crecimiento del CO y la FM. Se registró también un desplazamiento en sentido posterior del $\mathrm{CO}$, siendo este resultado inconsistente con lo hallado por LeCornu et al., (8). Esta inconsistencia podría estar relacionada al efecto biomecánico del Forsus, ya que al ser instalado el paciente sigue manteniendo su intercuspidación dentaria inicial.

Lima (22), analizó los cambios morfológicos del $\mathrm{CO}$ y de la FM en una muestra de 22 pacientes. En la muestra, 7 pacientes usaron AH y 15 usaron BG. En el grupo con $\mathrm{BG}$ el crecimiento del $\mathrm{CO}$ derecho fue de 6,12 $\mathrm{mm}$ y del CO izquierdo fue de $6,34 \mathrm{~mm}$. En el grupo con $\mathrm{AH}$ el crecimiento del $\mathrm{CO}$ derecho fue de 7,01 $\mathrm{mm}$ y del $\mathrm{CO}$ izquierdo fue de 6,84 $\mathrm{mm}$. No se encontró diferencia significativa en el crecimiento del $\mathrm{CO}$ entre los grupos que usaron $\mathrm{AH}$ y BG. La diferencia numérica entre las muestras (AH y BG) y la falta de un grupo control sin tratamiento podría significar una limitación de este estudio. Considerando que en la actualidad todavía existe reducida cantidad de trabajos con metodologías validadas de superposiciones de imágenes $3 \mathrm{D}$, este resultado tiene relevancia (figura 2).

Yildirim et al., (9), realizaron un estudio retrospectivo en registros de pacientes que usaron BG. Encontraron un crecimiento en dirección superior y posterior del CO. La muestra estuvo constituida por 30 pacientes y no hubo grupo control. La metodología fue calcular el volumen del CO para identificar cantidad de crecimiento o remodelación ósea. Bowen et al., (18), evaluaron a 20 pacientes que usaron BG por un tiempo de 12 meses. Encontraron un aumento en altura del CO de 6,20 $\pm 0,61 \mathrm{~mm}$ y un aumento anteroposterior de 7,22 $\pm 0,84 \mathrm{~mm}$.

Lima(22), Yildirim et al., (9) y Bowen et al., (18) utilizaron en sus investigaciones al aparato funcional BG y a la TCHC como herramienta de evaluación. En los tres estudios los resultados fueron coincidentes con un crecimiento del $\mathrm{CO}$, pero ningún estudio tuvo dentro de su respectiva metodología a un grupo control sin tratamiento ortodóncico, por lo que podemos sugerir que los resultados también fueron influenciados por el crecimiento de los pacientes (Tabla 1).

\section{Efectos sobre el Cóndilo y la Fosa Mandibular en el tratamiento de la maloclusión esquelética Clase III analizados con TC y TCHC}

Los efectos sobre el CO y la FM durante el tratamiento de la maloclusión esquelética Clase III también han sido analizados con la TCHC. En esta revisión de literatura, el protocolo de tratamiento frecuentemente encontrado fue el propuesto por De Clerck et al., (20). Cevidanes et al., (23), después de analizar a 21 pacientes que recibieron cirugía bimaxilar, encontraron un desplazamiento en dirección posterior del $\mathrm{CO}$ de $0,77 \mathrm{~mm}$. Otro grupo que recibió solo cirugía mandibular encontraron un desplazamiento posterior del $\mathrm{CO}$ de $0,7 \mathrm{~mm}$. No encontraron una diferencia significativa entre ambos grupos con relación desplazamiento del CO.

De Clerck et al., (11), en un estudio sobre 25 pacientes que recibieron un tratamiento de protracción maxilar con anclaje esquelético, encontraron un desplazamiento en dirección posterior del $\mathrm{CO}$ de $2,07 \mathrm{~mm}$. Estos resultados fueron consistentes con los encontrados por Nguyen et al., (12), quienes identificaron un desplazamiento en dirección posterior del $\mathrm{CO}$ de $2,6 \pm 1,5 \mathrm{~mm}$, también en 25 pacientes y con el mismo protocolo de tratamiento. Cabe resaltar que en ambos trabajos no se usaron grupo control, por lo que no se podría descartar el 
efecto del crecimiento en estos resultados, ya que en ambos trabajos los grupos tenían de 9 a 10 años de edad.

De Clerck et al., (11), también encontraron una remodelación ósea en la FM, con aposición en la parte anterior de 1,38 $\mathrm{mm}$ y reabsorción en la parte posterior de $1,34 \mathrm{~mm}$. Estos resultados hicieron pensar a los autores que podría existir una alta correlación entre el desplazamiento del CO y la remodelación de la FM cuando los pacientes fueron tratados con el respectivo protocolo de protracción maxilar (20).

Yatabe et al.,(13), estudiaron a 19 pacientes con Labio y Paladar Fisurado (LPF), quienes recibieron el protocolo de tratamiento propuesto por De Clerck (20). El grupo control no tuvo LPF pero si una maloclusión esquelética Clase III. Encontraron un desplazamiento en dirección posterior, lateral e inferior de la FM en ambos grupos. Los cambios esqueléticos en los pacientes con LPF fueron menores que los del grupo control. Podría sugerirse que la falta de consistencia ósea del maxilar en el grupo con LPF tendría alguna influencia en el menor respuesta ósea (Tabla 2).

En la búsqueda bibliográfica realizada, fueron pocos los estudios con TC y TCHC que analizaron al $\mathrm{CO}$ y la FM, lo cual podría considerarse como una limitación de la presente revisión de literatura. De los 13 estudios descritos anteriormente, solo 2 estudios tuvieron grupo control sin tratamiento ortodóncico alguno, 5 estudios tuvieron un grupo control con algún tratamiento alternativo y 6 estudios no tuvieron ningún grupo control. Sugerimos que la presencia o ausencia del grupo control podría estar influenciado por el factor ético, ya que irradiar a personas con crecimiento que no van a ser tratados ortopédicamente, científicamente es cuestionable.

El objetivo de la presente revisión de literatura fue identificar los cambios morfológicos del $\mathrm{CO}$ y FM por medio de modernas herramientas como la TCHC. Ante la escasez de estudios publicados en los últimos 10 años con relación al tema específico, se determinó como filtro de búsqueda 20 años de antigüedad de los artículos incluyéndose a las TC; es así como se incluyó al estudio de Croft et al., (5).

Palomino et al., (24), realizaron una revisión de literatura sobre los efectos de los aparatos propulsores de la mandíbula sobre la ATM, incluyó como criterios inclusión: estudios en animales, estudios con radiografías, evaluaciones con resonancia nuclear magnética y tomografías. Sólo reportaron dos estudios con tomografías y sin valores cuantitativos de los cambios del CO y de la FM. Por lo que nuestra revisión podría ser un buen complemento al estudio previo realizado.

Esta revisión de literatura fue realizada siguiendo una metodología integrativa la cual tuvo los siguientes pasos: (A) Identificación del problema, donde el objetivo de la búsqueda bibliográfica quedo definido; (B) búsqueda bibliográfica, en la cual se estableció una estrategia de selección de artículos aplicando los criterios de selección; (C) descripción de los datos, donde nos concentramos en la metodología utilizada; (D) análisis de los datos, donde hicimos las comparaciones de los resultados encontrados, y finalmente (E) presentación, en la cual se sintetiza toda la información obtenida durante el proceso de revisión de literatura para ser mostrada comprensivamente, destacando la importancia clínica así como las limitaciones que se tuvo en la revisión $(14,25)$.

\section{CONCLUSIONES}

En pacientes con maloclusión esquelética Clase II tratados con $\mathrm{AH}$ y $\mathrm{BG}$ se encontraron crecimiento en dirección posterior y superior del $\mathrm{CO}$ y un desplazamiento en dirección anterior de la FM y el CO. En pacientes con maloclusión esquelética Clase III tratados con protracción maxilar y anclaje esquelético, se encontró un desplazamiento en dirección posterior del CO y la FM, conjuntamente con una remodelación ósea en la FM. Esta remodelación ósea fue de tipo aposición en la región anterior y de tipo reabsorción en la región posterior de la FM. Las alteraciones se presentaron como crecimiento, remodelaciones óseas y desplazamientos.

La TC y la TCHC permiten conocer de manera cuantitativa y cualitativa los efectos sobre el CO y la FM cuando se usan aparatos con fuerzas ortopédicas.

\section{REFERENCIAS BIBLIOGRAFICAS}

1. Proffit WR, Field HW, Moray LJ. Prevalence of malocclusion and orthodontic treatment need in the United States: estimates from the N-HANES IIIsurvey. 
Int J Adult Orthod Otho Surg. 1998;13(2):97-106.

2. McNamara JA, Bryan FA. Long-term mandibular adaptations to protrusive function: an experimental study in Macaca mulatta. Am J Orthod Dentofacial Orthop. 1987;92(2):98-108.

3. Sato S, Susumu A, Tanaka EM. New concepts and treatment approach to the different types of malocclusions. Bull of Anagawa dent Col. 2007;35(1):48-125.

4. Aidar LA, Abrahão M, Yamashita HK, Dominguez GC. Morphological changes of condyles and Helkimo clinical dysfunction index in patients treated with herbst - Orthodontic appliance. Braz Dent J. 2013;24(4):31321.

5. Croft RS, Buschang PH, English JD, Meyer R. A cephalometric and tomographic evaluation of Herbst treatment in the mixed dentition. Am J Orthod Dentofacial Orthop. 1999;116(4):435-43.

6. Arici S, Akan H, Yakubov K, Arici N. Effects of fixed functional appliance treatment on the temporomandibular joint. Am J Orthod Dentofac Orthop. 2008;133(6):809-14.

7. Cevidanes LHC, Heymann G, Cornelis MA, DeClerck HJ, Tulloch JFC. Superimposition of 3-dimensional cone-beam computed tomography models of growing patients. Am J Orthod Dentofac Orthop. 2009;136(1):94-9.

8. Lecornu M, Cevidanes LHS, Zhu H, Wu CH, Larson B, Nguyen T. Three-dimensional treatment outcomes in Class II patients treated with the Herbst appliance: A pilot study. Am J Orthod Dentofac Orthop. 2013;144(6):81830.

9. Yildirim E, Karacay S, Erkan M. Condylar response to functional therapy with Twin-Block as shown by cone-beam computed tomography. Angle Orthod. 2014 ; 84(6):1018-25.

10. Cevidanes LHS, Styner MA, Proffit WR. Image analysis and superimposition of 3-dimensional conebeam computed tomography models. Am J Orthod Dentofac Orthop. 2006;129(5):611-8.

11. De Clerck H, Nguyen T, De Paula LK, Cevidanes LH. Three-dimensional assessment of mandibular and glenoid fossa changes after bone-anchored Class III intermaxillary traction. Am J Orthod Dentofac Orthop. 2012;142(1):25-31.

12. Nguyen T, Cevidanes L, Paniagua B, Zhu H, Koerich L, De Clerck H. Use of shape correspondence analysis to quantify skeletal changes associated with bone-anchored Class III correction. Angle Orthod. 2014;84(2):329-36.

13. Yatabe M, Garib D, Faco R, et al. Mandibular and glenoid fossa changes after bone-anchored maxillary protraction therapy in patients with UCLP: A 3-D preliminary assessment. Angle Orthod. 2017;87(3):42331.

14. Whittemore R, Knafl $K$. The integrative review: updated methodology. J Adv Nurs. 2005;52(5):546-53.

15. Al-Saleh MAQ, Alsufyani N, Flores-Mir C, Nebbe B, Major PW. Changes in temporomandibular joint morphology in class II patients treated with fixed mandibular repositioning and evaluated through 3D imaging: A systematic review. Orthod Craniofacial Res. 2015;18(4):185-201.

16. Souki BQ, Vilefort PLC, Oliveira DD, et al. Threedimensional skeletal mandibular changes associated with Herbst appliance treatment. Orthod Craniofacial Res. 2017;20(2):111-8.

17. Batista KB dos SL, Lima T, Palomares N, Carvalho F de $\mathrm{A}$, et al. Herbst appliance with skeletal anchorage versus dental anchorage in adolescents with Class II malocclusion: Study protocol for a randomised controlled trial. Trials. 2017;18(1):1-11.

18. Bowen L, Yanmin W, Fang S, Min L, Ying D, Li Z. Analysis of temporomandibular joint changes before and after Twin-block correction by cone-beam CT analysis of Class II division 1 malocclusion. West China of Stomatology. 2013;31(6):610-4.

19. Santamaría-Villegas A, Manrique-Hernandez R, Alvarez-Varela E, Restrepo-Serna C. Effect of removable functional appliances on mandibular length in patients with class II with retrognathism: Systematic review and meta-analysis. BMC Oral Health. 2017;17(1):1-9.

20. De Clerck HJ, Cornelis MA, Cevidanes LH, Heymann GC, Tulloch CJF. Orthopedic traction of the maxilla with miniplates: A new perspective for treatment of midface deficiency. J Oral Maxillofac Surg. 2009 ; 67(10):2123-9.

21. Bjork A, Skieller V. Normal and abnormal growth of the mandible. A synthesis of longitudinal cephalometric implant studies over a period of 25 years. Eur J Orthod. 1983;5(1): $1-46$.

22. Lima T. Three-dimensional assesment of dentoskeletal mandibular changes resulting from treatment with Herbts and Twin Block appliances. Tesis de Grado PhD. Rio de Janeiro: Rio de Janeiro State University. 2016. $111 \mathrm{pp}$.

23. Cevidanes LHS, Bailey LJ, Tucker SF, et al. Threedimensional cone-beam computed tomography for assessment of mandibular changes after orthognathic surgery. Am J Orthod Dentofac Orthop. 2007;131(1):4450.

24. Palomino S, Almeida KM, de Mello PB, Restrepo M, Ravelr DB. CES odontología. 2014; 27: 82-92 p.

25. Hopia A, Latvala E, Liimatainen L. Reviewing the methodology of an integrative review. Scand J Caring Sci. 2016;30(4):662-9.

Recibido : 29-01-2021

Aceptado : 22-04-2021 